\title{
A Comparative Study to Assess the Efficacy of Streptokinase in Diabetic Versus Non-Diabetic Acute ST Elevation Myocardial Infarction Patients
}

\section{Zeeshan Hassan, ${ }^{1}$ Nabeegh Rana, ${ }^{2}$ Bakhtawar Rana, ${ }^{3}$ Asif Iqbal, ${ }^{4}$ Ali Javaid Chughtai ${ }^{5}$}

\begin{abstract}
Objective: Prominent resolution in the ST segment elevation on electrocardiogram(ECG), thrombolysis at the infarction site restoring perfusion determines the effectiveness of the streptokinase therapy. Hypercoagulable states and lack of efficacy with streptokinase is seen in diabetics. This study aimed to assess the thrombolytic efficacy of streptokinase in diabetic vs non-diabetics patients.

Methods: A cross-sectional study was conducted at Cardiology Department of Allama Iqbal Memorial Teaching Hospital, Sialkot from 1st September 2019 to 30th April, 2020. Total 504 patients of which 185 diabetics and 319 non-diabetic were selected. All the patients presenting with first episode of acute STelevation myocardial infarction were thrombolysed with 1.5 million units of streptokinase within 12 hours from the onset of their typical chest pain symptoms. A complete record of ECG changes was kept before and $90 \mathrm{~min}$ after thrombolysis with streptokinase. Chi- square test was applied and $p$ value $<0.05$ was considered significant.

Results: $89.19 \%$ diabetic patients had $>70 \%$ resolution of ST segment changes in comparison to $95.61 \%$ non-diabetics. $16.76 \%$ of the diabetic patients had increased ST-segment elevation post thrombolysis (Pvalue 0.001 ). $8.11 \%$ and $10.81 \%$ reinfarction rates during hospital stay and at one month post-thrombolysis were recorded in diabetics. Reduced left ventricle Ejection Fraction was seen in $62.16 \%$ and $58.62 \%$ of the diabetic and non-diabetic patients $(\mathrm{P}$-value $<0.005)$.

Conclusion: Comparatively decreased efficacy of streptokinase is seen in diabetic patients with reduced resolution of ST-segment. In correspondence with reduced left ventricle EF, re-infarction and stroke episodes.

Key Words: Streptokinase, acute myocardial infarction, STEMI, diabetes mellitus, hypercoagulability, atherosclerosis.

How to Cite: Hassan Z., Rana N., Rana B., Iqbal I., Chughtai J.I. A comparative study to assess the efficacy of streptokinase in diabetic versus non-diabetic acute ST elevation myocardial infarction patients. Esculapio 2021;17(01):88-92

DOI: https://doi.org/10.51273/esc21.2517118
\end{abstract}

\section{Introduction}

$\mathrm{I}$ $\mathrm{n}$ Pakistan, one of the major cause of increasing mortality and morbidity is myocardial infarction

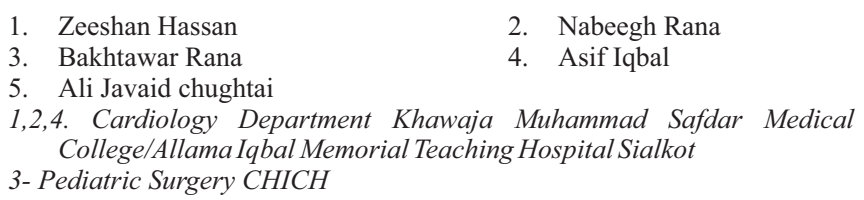

Correspondence:

Dr. Zeeshan Hassan, Cardiology Department Khawaja Muhammad Safdar Medical College/Allama Iqbal Memorial Teaching Hospital Sialkot Email:Dr.zee_196@hotmail.com mainly due to atherosclerosis of the coronary arteries. ${ }^{1}$ Acute myocardial infarction has mainly increased the death ratio in many world leading countries. ${ }^{2}$ Majority of the population in Pakistan between 46-55 years of age are at risk of myocardial infarction. ${ }^{3}$

Diabetes mellitus is one of the main co-morbidities associated with myocardial infarction others being dyslipidemia, smoking, hypertension and previous episode of ischemic heart diseases. ${ }^{4}$ High blood sugar or glucose levels in diabetics identifying poor glycemic control are important cause of hypercoagulable states in diabetic patients. ${ }^{5}$ Therefore, hypercoagulable state risk is considered more common in diabetic population. ${ }^{6}$ Also fibrinolysis is deceased in diabetics 
as a result of increased plasminogen activator inhibitor type 1, thus progressive thrombus formation and accelerated plaque formation. ${ }^{7}$ This increases the incidence of myocardial infarction among diabetics as compared to non-diabetics. ST-segment resolution is a predictor to see the outcomes in myocardial infarction patients. ${ }^{8}$ Early thrombolysis with streptokinase in acute stage of myocardial infarction to restore blood supply of the myocardial cells is the main goal. Significant ST segment changes on ECG after thrombolytic therapy is more economical for accessing the prognosis of the disease, comparatively to coronary angiogram. Increased mortality and morbi-dity is observed widely in diabetic patients post thrombolysis.'

Our study mainly aimed at assessing the efficacy and outcomes in diabetic patients receiving thrombolytic therapy with streptokinase in acute myocardial infarction and comparing the results with non-diabetic patients. The efficacy of streptokinase associated with diabetic population will be assessed by electrical parameters (ECG changes) $>70 \%$ or $<70 \%$ ST-segment resolution, relieve of typical chest-pain symptoms, raised CK-MB levels, number of re-infarctions or stroke episodes and left ventricle ejection fraction (E.F\%) estimation through echocardiography postthrombolysis. This will help choose different treatment options as coronary interventions in diabetic patients with associated morbidities especially acute myocardial infarction.

\section{Methods}

A cross-sectional study was conducted at the department of cardiology, Allama Iqbal Memorial Teaching Hospital, Sialkot. The data was collected over a period of eight months from 1st September, 2019 till 30th April , 2020 through electronic databases. After informed consent, 504 patients were carefully registered of which 185 were diabetics and 319 non-diabetics. After confirmation their data (including their complete medical or any surgical history) was entered on the computerized database. The inclusion criteria had patients (diabetics or non-diabetics) with first episode of MI, suffering from typical chest-pain for $>30$ mins but $<12$ hours, ST-segment elevation in concordant leads on ECG and were thrombolysed with streptokinase within 12 hours from onset of their symptoms. Pregnant females, those patients with any contraindications to thrombolysis with streptokinase, other than ST-segment ECG changes, with chest pain for $>12$ hours, having previous cardiac intervention or history of ischemic heart disease were in the exclusion criteria. The patients were divided into two groups i-e diabetics and non-diabetics. Only previously diagnosed diabetic patients (HbA1C levels $>6.5 \%$ ) were enrolled. The study is approved by research and ethics committee of KMSMC/AIMTH, Sialkot letter No.46/REC/KMSMC Date:04/01/2020.

An ECG was obtained on presentation and 90 minutes after streptokinase therapy for complete record. The outcome of thrombolysis with streptokinase in both the groups was assessed based on electrical parameters, relieve of patient symptoms, tachycardia episodes, reduction in left ventricle systolic function by calculating $\mathrm{EF}(\%)$ on bedside echocardiography and post thrombolysis reinfarction or stroke episodes. The electrical parameters were assessed whether the patient had increased or persistent ST segment elevation, or had $>70 \%$ decrease / resolution in ST segment elevation after administration of streptokinase, which is considered to be a successful reperfusion sign. ECG reporting was done by a single senior E.R doctor to avoid any discrepancies in interpretation. Similarly, continuous monitoring for any re-infarction (with raised Ck-MB levels or new concordant ST-segment elevations on ECG within 6 days of patients hospital stay) was done. Episodes of cerebrovascular accidents of the same patients were recorded easily due to readmission of the patients to the same hospital. Regular follow-ups of all the enrolled patients were ensured every 1 month for first six months after their acute MI episode by repeated telephonic reminders and than updating their data on electronic data collection devices respectively.

The data were analyzed by using SPSS version 23 . Mean and standard deviation were used to calculate quantitative variables as age. Both (male $\&$ female ) genders were included as qualitative variables and were measured using percentages. Outcome variables for successful reperfusion were recorded and the results compared between diabetics and non-diabetics groups using Chi-square test. $\mathrm{P}$-value of $<0.05$ was considered significant.

\section{Results}

A total of 504 patients were enrolled, of which 185 
were diabetics and 319 non-diabetics, mean age in the diabetic group was $54 \pm 9$.6yrs as compared to $57 \pm$ $13.3 \mathrm{yrs}$ in non-diabetics (table 1). Age range was between 44 to $64 \mathrm{yrs}$ in diabetics and 42 to $71 \mathrm{yrs}$ in the non-diabetic group. Among the diabetic group 90 (48.65\%) were males and 95 (51.35\%) were females whereas, the other comparative group of non-diabetics had greater number of males $232(72.72 \%)$ than $87(27.27 \%)$ females with the significant P-value of 0.001 . Average basal metabolic index (BMI) of $33.71 \pm 9$ was calculated in diabetic population and $23.51 \pm 7.5$ in non-diabetic group. According to the

Table 1: Baseline Characteristics of Patients

\begin{tabular}{lccc}
\hline & Diabetics & Non-diabetics & p-value \\
\hline AGE & $54 \pm 9.6(34.3 \%)$ & $57 \pm 13.3(65.7 \%)$ & .082 \\
GENDER & & & \\
Male & $90(48.65 \%)$ & $232(72.72 \%)$ & .001 \\
Female & $95(51.35 \%)$ & $87(27.27 \%)$ & \\
BMI & $33.71 \pm 9(37.8 \%)$ & $23.51 \pm 7.5(62.2 \%)$ & .603 \\
Cholesterol & $185.19 \pm 37.52$ & $170.81 \pm 38.34$ & .037 \\
& $(35.0 \%)$ & $(65.0 \%)$ & \\
HbA1c & $9.65 \pm 2.66$ & $6.01 \pm 0.76$ & .008 \\
& $(46.4 \%)$ & $(53.6 \%)$ & \\
\hline
\end{tabular}

Table 2: Post Streptokinase Therapy Efficacy Determinants

\begin{tabular}{|c|c|c|c|c|c|}
\hline & \multicolumn{2}{|c|}{ Diabetics } & \multicolumn{2}{|c|}{ Non-Diabetcs } & \multirow[t]{2}{*}{$\begin{array}{c}\text { P- } \\
\text { Value }\end{array}$} \\
\hline & count & $\%$ & count & $\%$ & \\
\hline Symptoms relieved & 98 & $52.97 \%$ & 258 & $80.88 \%$ & .004 \\
\hline $\begin{array}{l}\text { Inhospital } \\
\text { rethrombolysis }\end{array}$ & 15 & $8.11 \%$ & 10 & $3.13 \%$ & .000 \\
\hline One month reinfarction & 20 & $10.81 \%$ & 15 & $4.70 \%$ & .004 \\
\hline One month stroke & 3 & $5.0 \%$ & 0 & $0.0 \%$ & .041 \\
\hline Six month reinfection & 5 & $2.70 \%$ & 0 & $0.0 \%$ & .280 \\
\hline Six month stroke & 2 & $1.08 \%$ & 0 & $0.0 \%$ & .447 \\
\hline $\begin{array}{l}\text { St-Segment Elevation } \\
\text { increased }\end{array}$ & 31 & $16.76 \%$ & 0 & $0.0 \%$ & \\
\hline $\begin{array}{l}\text { St-Segment } \\
\text { Resolution }>70 \%\end{array}$ & 165 & $89.19 \%$ & 305 & $95.61 \%$ & .001 \\
\hline $\begin{array}{l}\text { St-segment } \\
\text { Resolution }<70 \%\end{array}$ & 20 & $10.81 \%$ & 14 & $4.39 \%$ & \\
\hline CK-MB (raised) & 8 & $4.32 \%$ & 7 & $2.19 \%$ & 0.063 \\
\hline Heart rate $>100 \mathrm{bpm}$ & 80 & $43.24 \%$ & 105 & $32.92 \%$ & 0.013 \\
\hline $\begin{array}{l}\text { Reduced LV ejection } \\
\text { fraction }\end{array}$ & 115 & $62.16 \%$ & 187 & $58.62 \%$ & 0.000 \\
\hline
\end{tabular}

biochemical parameters significantly increased $\mathrm{HbA} 1 \mathrm{c}$ values $9.65 \pm 2.66$ were seen in the diabetics with $\mathrm{P}$ value 0.008 . Blood cholesterol levels were found deranged among the two groups. (table 1)
$52.97 \%$ of the diabetics and $80.88 \%$ non-diabetics had their symptoms relieved with a significant Pvalue 0.004 (table.2). The incidence of re-infarction in diabetics during their hospital stay ( $<6$ days) was $8.11 \%$ (P-value 0.000$)$ and $10.81 \%(\mathrm{P}$-value .004) after one month. With regards to the electrical activity our results showed that $16.76 \%$ (P-value 0.001 ) of diabetics showed increased or persistent ST segment elevation. $95.61 \%$ of the non-diabetics in comparison to diabetics $89.19 \%$ had $>70 \%$ reduction in the STsegment post thrombolysis(P-value 0.001). About $10.81 \%$ diabetics in group 1 had $<70 \%$ ST segment resolution after thrombolytic therapy. $43.24 \%$ diabetics had episodes of tachycardia (H.R $>100 \mathrm{bpm}$ ) and $62.16 \%$ ( $p$ value $<0.005$ ) had reduced left ventricular ejection fraction in comparison to $58.62 \%$ nondiabetic MI patients(table 2).

\section{Discussion}

Thrombolysis with streptokinase is an effective and well known treatment option in patients presenting with acute ST-elevation myocardial infarction. Presence of associated co-morbidities or late ( $>12$ hours) presentation can affect the efficacy of streptokinase therapy 10. All the patients in our study presented in $>30$ mis from the onset of their symptoms but not more than 12 hours. Diabetes is one of the main risk factors associated with cardiovascular diseases. ${ }^{11}$ Increased morbidity is associated with diabetic acute MI patients and reduce effect of streptokinase was seen in a research by varshit hathi. ${ }^{12}$ This led us to make a hypothesis that diabetes solely may effect the efficacy and the outcomes of streptokinase therapy. Therefore, we conducted this study to assess the efficacy of streptokinase and compared the results specifically among diabetic and non-diabetic groups with acute ST-elevation myocardial infarction.

The mean HbA1c levels in diabetic population were $9.65 \pm 2.66$ and $6.01 \pm 0.76$ in non-diabetic group. HbA1c is one of the important biomarkers to diagnose diabetes and to monitor its prognosis. ${ }^{13}$ Therefore, $\mathrm{HbA} 1 \mathrm{c}$ is used as continuous risk measurement variable for coronary artery diseases. Post thrombolysis reduced or decreased ST-segment resolution is seen significantly in diabetic patients in comparison to nondiabetics. ${ }^{14}$ This was quite similar to our study results where comparatively reduced ST-segment resolution was seen in the diabetic group, $10.81 \%$ of the diabetics had $<70 \%$ resolution of ST-segment and $16.76 \%$ 
showed persistently increased ST-segment elevations post thrombolysis (P-value 0.001). Significant resolution of ST-segment was seen in $95.61 \%$ non-diabetic group (P-value 0.001 ) with no persistent or increase elevations pot thrombolysis.

Among diabetic patients 98(52.97\%) had their typical chest pain symptoms relieved after thrombolysis with streptokinase whereas 258 out of 319 non-diabetics patients had their typical chest pain symptoms relieved with a significant $p$-value (0.004). $8.11 \%$ of the diabetics had re-infarction during their hospital stay and were re-thrombolysed with streptokinase in comparison to only $3.13 \%$ non-diabetics. According to our results the risk of stroke at one month in diabetics was $5 \%$ and $1.08 \%$ at six months with no episodes seen in the other comparative group. So was the risk of six month re-infarction which was common in diabetics. These results were consistent with the Framingham heart study, which proved after 20 years of trials that diabetic patients have two-fold to three-fold increased risk of clinical atherosclerosis and the cardiovascular disease mortality was greater for diabetic women as compared to diabetic men but after adjustment of other associated risk factors it was same for men and women. ${ }^{15}$

Similarly, other determinants as heart rate and effect on left ventricle ejection fraction after streptokinase therapy were assessed. Some researchers stated that patients with diabetes respond poorly after thrombolytic therapy. ${ }^{16}$ As was seen in our study results $43.24 \%$ diabetics had episodes of tachycardia (heart rate $>100 \mathrm{bpm}$ ) and most of them $62.16 \%$ had reduction in their left ventricle ejection fraction in comparison to $58.62 \%$ non diabetic patients. The limitations to our study included that we collected data from only one center with patients belonging to same ethnic backgrounds. Moreover, poor compliance of patients with medication and sedentary life styles were noticed. Therefore, more attention is required for treating diabetic myocardial infarction patients. Other treatment options as coronary interventions may be considered in diabetic acute STEMI patients.

\section{Conclusion}

In comparison to non-diabetics, decrease efficacy of streptokinase is seen in diabetic patients with reduced resolution of ST-segment .Post thrombolysis failure was correlated with reduced EF, re-infarction and stroke episodes.

\section{Conflict of Interest: $\quad$ None}

\section{References}

1. Haq H, Kiyani AM. The prevalence of hyperlipidemia in patients suffering from coronary artery disease. Pakistan Heart Journal. 2012 Jul 26;32(3).

2. Balda-Canizares JA, Tamariz L, Moreno-Zambrano D, Pareja D, Ortiz-Prado E, Palacio A, Palacio A. Increasing myocardial infarction mortality trends in a middle-income country. Cardiovascular diagnosis and therapy. 2018 Aug;8(4):493.

3. Karim MA, Qureshi AA, Mahmood SF, Akhter J. Thrombolytic therapy in acute myocardial infarction in Pakistan. JPMA. The Journal of the Pakistan Medical Association. 1995;45(3):54.

4. Gheisari F, Emami M, Raeisi Shahraki H, Samipour $\mathrm{S}$, Nematollahi P. The Role of Gender in the Importance of Risk Factors for Coronary Artery Disease. Cardiology Research and Practice. 2020 Jul 29;2020.

5. Kadiyala SR, Rao K, Rao NR, Bhat R, Rao J, Navin P, Balaji O. Association of postprandial blood sugar with hypercoagulability in comparison to fasting blood sugars in diabetic and healthy patients: A crosssectional study. Asian Journal of Pharmaceutical and Clinical Research. 2017 Jan 1;10(7):378-84.

6. Pomero F, Di Minno MN, Fenoglio L, Gianni M, Ageno W, Dentali F. Is diabetes a hypercoagulable state? A critical appraisal. Acta diabetologica. 2015 Dec; 52(6):1007-16.

7. Almourani R, Chinnakotla B, Patel R, Kurukulasuriya LR, Sowers J. Diabetes and cardiovascular disease: an update. Current diabetes reports. 2019 Dec; 19(12):1-3.

8. Spitaleri G, Brugaletta S, Scalone G, Moscarella E, Ortega-Paz L, Pernigotti A, Gomez-Lara J, Cequier A, Iñiguez A, Serra A, Jiménez-Quevedo P. Role of ST-segment resolution in patients with ST-segment elevation myocardial infarction treated with primary percutaneous coronary intervention (from the 5-Year Outcomes of the EXAMINATION [Evaluation of the Xience-V Stent in Acute Myocardial Infarction] Trial). The American journal of cardiology. 2018 May 1;121(9):1039-45.

9. Rosano GM, Vitale C, Seferovic P. Heart failure in patients with diabetes mellitus. Cardiac failure review. 2017Apr;3(1):52.

10. Saleem $S$, KhanA, Shafiq I. Post thrombolytic resolution of ST elevation in STEMI patients. Pakistan journal of medical sciences. 2016 Jan;32(1):201.

11. Leon BM, Maddox TM. Diabetes and cardiovascular disease: Epidemiology, biological mechanisms, treatment recommendations and future research. World 
journal of diabetes. 2015 Oct 10;6(13):1246.

12. Hathi V, Anadkat M. A Comparative Study of InHospital Outcome of Patients with ST-Segment Elevation Myocardial Infarction with and Without Diabetes Mellitus, after Thrombolytic Therapy; In Government Hospital of Rajkot, Gujarat, India. The Journal of the Association of Physicians of India. 2017 Nov 1;65(11):22-5.

13. Sherwani SI, Khan HA, Ekhzaimy A, Masood A, Sakharkar MK. Significance of HbA1c test in diagnosis and prognosis of diabetic patients. Biomarker insights. 2016 Jan;11:BMI-S38440.

14. Iqbal S, Bari MS, Bari MA, Islam MM, Majumder MA, Islam Z, Aditya GP, Paul GK, Shakil SS, Saha B, Paul PK. A comparative study of ST segment resolution between diabetic and non-diabetic ST segment elevation myocardial infarction patients following streptokinase thrombolysis. Cardiovascular Journal. 2019 Feb 27;11(2):118-22.

15. Kannel WB. Framingham study insights on diabetes and cardiovascular disease. Clinical chemistry. 2011 Feb 1;57(2):338-9.

16. Saleem S, Khan A, Shafiq I. Post thrombolytic resolution of ST elevation in STEMI patients. Pakistan journal of medical sciences. 2016 Jan;32(1):201.
Authors Contribution
HZ: Conceptionlization of Project
RB: Data Collection
RN: Literature Search
IA,RN,CJI: Statistical Analysis
RN: Drafting, Revision
RN: Writing of Manuscript 